\title{
Condição ocular dos indivíduos facectomizados na região centro-oeste do estado de São Paulo: estudo populacional
}

\author{
Cataract-operated individuals at the Center-Western zone of São Paulo state: \\ populational survey
}

\author{
Olívia Matai ${ }^{1}$ \\ Silvana Artioli Schellini ${ }^{2}$ \\ Carlos Roberto Padovani ${ }^{3}$
}

Trabalho realizado na Faculdade de Medicina de Botucatu - UNESP - Botucatu (SP) - Brasil.

${ }^{1}$ Residente de 3a ano de Oftalmologia do Departamento de Oftalmologia, Otorrinolaringologia e Cirurgia de $\mathrm{Ca}$ beça e Pescoço da Faculdade de Medicina da Universidade Estadual Paulista "Júlio Mesquita Filho" UNESP - Botucatu (SP) - Brasil.

${ }^{2}$ Professora Livre-docente do Departamento de Oftalmologia, Otorrinolaringologia e Cirurgia de Cabeça e Pescoço da UNESP - Botucatu (SP) - Brasil.

${ }^{3}$ Professor Titular do Departamento de Bioestatística do Instituto de Biociências da UNESP - Botucatu (SP) Brasil.

Endereço para correspondência: Silvana Artioli Schellini - Depto de OFT/ORL/CCP. Faculdade de Medicina de Botucatu-UNESP - Botucatu (SP)

CEP 18618-000

E-mail:sartioli@fmb.unesp.br

Recebido para publicação em 05.01.2007

Última versão recebida em 11.09.2007

Aprovação em 24.09.2007

Nota Editorial: Depois de concluída a análise do artigo sob sigilo editorial e com a anuência do Dr. Marinho Jorge Scarpi sobre a divulgação de seu nome como revisor, agradecemos sua participação neste processo.

\section{RESUMO}

Objetivo: Avaliar as causas de baixa visão e cegueira em indivíduos facectomizados, de amostra da população de cidades da região centrooeste do estado de São Paulo. Métodos: Estudo transversal, observacional, feito em cinco cidades da região centro-oeste do estado de São Paulo, em amostra domiciliar e baseada nos dados do último Censo Demográfico (IBGE, 1995), com escolha sistemática dos domicílios. Foi considerada para o presente estudo uma subamostra de indivíduos facectomizados, dos quais foram obtidos dados de identificação e exame oftalmológico completo. Os dados foram avaliados por estatísticas descritivas, análise de frequiência de ocorrência e proporção de concordância, com intervalo de confiança de 95\%. Resultados: Dos indivíduos amostrados, 2,37\% haviam sido submetidos à facectomia. Dos 201 olhos operados, 26,9\% apresentavam acuidade visual compatível com cegueira ou deficiência visual. Com a melhor correção óptica, a acuidade visual permaneceu $<0,3$ em 19,0\%. O exame refracional proporcionou melhora da acuidade visual para $27,9 \%$ dos indivíduos facectomizados. As causas de baixa visão foram os erros refrativos não corrigidos, opacidade de cápsula posterior $(19,4 \%)$, ceratopatia bolhosa $(8,3 \%)$ coriorretinite cicatricial $(8,3 \%)$, afacia $(8,3 \%)$, degeneração macular relacionada a idade $(5,5 \%)$, leucoma $(5,5 \%)$, glaucoma $(5,5 \%)$, atrofia de papila $(5,5, \%)$, descolamento de retina $(2,8 \%)$, atrofia de epitelio pigmentado da retina $(2,8 \%)$ e alta miopia (2,8\%). Conclusão: Apesar da catarata ser causa de cegueira que pode ser evitável, mesmo após a correção cirúrgica porcentagem expressiva de indivíduos permanece com baixa visão, em geral, em decorrência de fatores relacionados ao seguimento pós-operatório negligenciado.

Descritores: Extração de catarata; Promoção da saúde; Cegueira/prevenção \& controle; Baixa visão; Levantamentos demográficos; Serviços de saúde comunitária

\section{INTRODUÇÃO}

Em 1999, a Organização Mundial da Saúde (OMS) em conjunto com a Agência Internacional de Prevenção à Cegueira, criaram um programa intitulado "2020 - O Direito à Visão", que tem como objetivo chegar ao ano de 2020 sem cegos por motivos preveníveis ou tratáveis ${ }^{(1)}$.

Atualmente no Brasil há cerca de 4 milhões de deficientes visuais e 1.250.000 cegos, sendo que 70 a $80 \%$ das causas de cegueira são preveníveis ou tratáveis ${ }^{(2)}$. 
Isto representa não apenas um problema para os indivíduos afetados, mas também para o Estado, devido aos custos econômicos e sociais. Em vista disso, esforços têm sido feitos na tentativa de diminuir o número de casos de cegueira e deficiência visual que sejam decorrentes de afecções previníveis ou tratáveis, inclusive com grande apoio dos oftalmologistas e do Conselho Brasileiro de Oftalmologia.

No caso da catarata, a principal causa de cegueira em indivíduos maiores de 60 anos, a estratégia seria aumentar o número de cirurgias oferecidas à população ${ }^{(2)}$.

No Brasil, um dos locais onde a catarata ainda é um sério problema de saúde ocular, é indiscutível a necessidade de se aumentar o número de cirurgias de catarata e nos últimos anos esforços foram feitos para possibilitar maior acesso da população aos serviços gratuitos, tendo em vista que muitas das pessoas portadoras do problema seriam pessoas de baixa renda.

Entretanto, é igualmente importante avaliar a qualidade dos serviços que estão sendo oferecidos, tendo em vista que existe a necessidade de conhecer a real situação dos pacientes após o tratamento, a fim de verificar os resultados das ações que estão sendo realizadas na população.

Desta forma, o objetivo deste estudo foi avaliar o resultado e as causas de baixa visão e cegueira em indivíduos facectomizados, habitantes da região centro-oeste do estado de São Paulo.

\section{MÉTODOS}

Este foi um estudo transversal, de caráter observacional, realizado em cinco cidades localizadas na região centro-oeste do estado de São Paulo, a saber: Botucatu, Pratânia, Piraju, Areiópolis e Arandu. Foram incluídos no estudo apenas os moradores permanentes dos Municípios.

A amostra do estudo foi domiciliar, baseada nos setores censitários do Censo Demográfico (IBGE, 1995), obedecendo aleatorização por seleção da primeira casa por sorteio; depois disso, as demais casas foram escolhidas de forma sistemática, optando-se pela sexta casa localizada do lado par da rua e assim, sucessivamente. Caso a moradia sorteada estivesse desabitada, a primeira casa situada à direita da sorteada era considerada para o estudo. Caso esta também se encontrasse desabitada, a primeira casa da esquerda da casa inicialmente escolhida era considerada para o estudo.

Todos os membros de cada família sorteada foram convidados a comparecer, em data previamente marcada, para um exame oftalmológico que consistia em avaliação da acuidade visual (AV), tonometria, biomicroscopia, fundoscopia e exame refracional.

Os exames foram realizados usando a Unidade Móvel Oftalmológica da Faculdade de Medicina de Botucatu - UNESP, nos anos de 2005 e 2006.

No total, foram atendidos 5.555 indivíduos moradores destes Municípios, sendo que este número representou $78 \%$ da amostra pretendida.
Para o presente estudo foram analisados os dados de uma subamostra, constituída por indivíduos que haviam sido submetidos previamente à cirurgia de catarata, em um ou em ambos os olhos, com ou sem implante de lente intra-ocular, nos diversos serviços que ofereceram a cirurgia.

Os parâmetros avaliados foram: idade, sexo, acuidade visual antes e após correção óptica e diagnósticos associados.

Os dados pessoais e anamnese oftalmológica foram obtidos de cada participante, por meio de um protocolo que continha questões fechadas.

A acuidade visual (AV) foi obtida com a correção óptica em uso (quando presente) e também sem correção, tendo sido avaliada em cada olho separadamente, em ambiente iluminado e usando a tabela do E de Snellen, situada a 5 metros do paciente.

Em seguida, o paciente foi avaliado em auto-refrator e refração subjetiva, anotando-se a AV de cada olho resultante após o exame. A AV foi classificada em 4 categorias: $\mathrm{AV}>0,7 ; 0,3<\mathrm{AV}<0,7 ; 0,05<\mathrm{AV}<0,3$ e $\mathrm{AV}<0,05$. Considerou-se cego o olho com AV menor que 0,05 e como deficiente visual o olho com AV entre 0,3 e 0,05 , ou seja, o conceito de cegueira e deficiência visual adotado esteve relacionado ao olho e não ao indivíduo.

A correção óptica foi prescrita nos casos em que houve melhora da AV após a refração.

A biomicroscopia foi realizada em lâmpada de fenda (BQ-900, Haag Streit, Bern, Switzerland), seguida por fundoscopia usando lente de Volk 78D, após a dilatação da pupila com 3 gotas de colírio tropicamida $1 \%$.

Os dados coletados foram transferidos para planilha Excel e analisados segundo estatísticas descritivas, análise de freqüência de ocorrência e proporção de concordância, com intervalo de confiança de $95 \%$.

\section{RESULTADOS}

Dos 5.555 indivíduos atendidos pela unidade móvel nos municípios em questão, 2.291 (41,2\%) eram homens e 3.264 $(58,8 \%)$, mulheres. Destes, $1.599(28,78 \%)$ possuíam idade de 50 anos ou mais.

Avaliando-se os indivíduos que já haviam feito facectomia, $132(2,37 \%)$ haviam sido submetidos à cirurgia de catarata em um $(47,7 \%)$ ou em ambos $(52,3 \%)$ os olhos. Quanto ao sexo, $73(55,3 \%)$ eram mulheres e $59(44,7 \%)$ homens. Em relação à idade, a grande maioria, ou seja, 126 indivíduos $(95,4 \%)$ que foram operados de catarata apresentavam 50 anos ou mais.

Dos 201 olhos operados, 7 (3,48\%) permaneceram afácicos, sendo afácicos unilaterais 2 homens e 3 mulheres e outros 2 homens portadores de afacia bilateral, todos maiores que 70 anos. Após refração, os 2 afácicos bilaterais resultaram em AV entre 0,3 e 0,7. Outros 3 olhos de indivíduos portadores de afacia unilateral, tiveram AV com correção óptica menor que 0,05 .

A AV antes do exame de refração foi maior que 0,7 em 
apenas 83 (43,9\%) olhos; entre 0,3 e 0,7 em 55 (29,1\%); entre 0,05 e 0,3 em $27(14,2 \%)$ e menor que 0,05 em $24(12,7 \%)$ olhos. Ou seja, antes da correção óptica, 26,9\% dos indivíduos facectomizados apresentavam ainda acuidade visual compatível com cegueira ou deficiência visual (Tabela 1).

Após a refração, houve melhora da AV em 56 olhos (27,9\%). Com correção óptica, 119 (62,9\%) olhos obtiveram $\mathrm{AV}>0,7 ; 34(17,9 \%)$ AV entre 0,3 e 0,$7 ; 20$ (10,58\%) AV entre 0,05 e 0,3 e $16(8,46 \%) \mathrm{AV}<0,05$. Ou seja, mesmo com a melhor correção óptica, a AV permaneceu no nível de cegueira ou deficiência visual em 19,0\% dos olhos operados (Tabela 1).

A emetropia foi encontrada em 46 (26,3\%) olhos. Os erros refracionais encontrados foram: astigmatismo hipermetrópico $(27,4 \%)$, astigmatismo simples $(22,3 \%)$, astigmatismo miópico $(17,7 \%)$, miopia $(3,4 \%)$ e hipermetropia $(2,8 \%)$ (Tabela 2$)$.

Dos 36 olhos com AV menor que 0,3 e que não tiveram melhora com a correção óptica, $7(19,4 \%)$ apresentavam opacidade de cápsula posterior. Outros diagnósticos associados à baixa visão foram: ceratopatia bolhosa (3 olhos - 8,3\%), coriorretinite cicatricial ( 3 olhos $-8,3 \%$ ), afacia (3olhos - 8,3\%), degeneração macular relacionada à idade (2 olhos $-5,5 \%)$, leucoma ( 2 olhos - 5,5\%), glaucoma ( 2 olhos - 5,5\%), atrofia de papila (2 olhos - 5,5,\%), descolamento de retina (1 olho $-2,8 \%$ ), atrofia de epitélio pigmentado da retina ( 1 olho - $2,8 \%$ ) e alta miopia ( 1 olho - 2,8\%). Em 7 olhos a causa da baixa visão permaneceu indeterminada.

\section{DISCUSSÃO}

Nos últimos anos, esforços têm sido feitos na tentativa de aumentar o número de cirurgias de catarata, na tentativa de

\begin{tabular}{|c|c|c|c|c|c|}
\hline \multirow[t]{2}{*}{ Sem correção } & \multicolumn{5}{|c|}{ Com correção } \\
\hline & $>0,7$ & $0,3-0,7$ & $0,05-0,3$ & $<0,05$ & Total \\
\hline$>0,7$ & 81 & 2 & 0 & 0 & 83 \\
\hline $0,3-0,7$ & 36 & 19 & 0 & 0 & 55 \\
\hline $0,05-0,3$ & 1 & 12 & 14 & 0 & 27 \\
\hline$<0,05$ & 1 & 1 & 6 & 16 & 24 \\
\hline Total & 119 & 34 & 20 & 16 & 189 \\
\hline
\end{tabular}

reduzir a cegueira prevenível decorrente desta causa. No ano de 2006, estima-se que 360 mil cirurgias tenham sido realizadas no Brasil (2).

Entretanto, deve-se buscar a melhor qualidade dos serviços prestados aos portadores de catarata, uma vez que não basta o ato operatório executado com técnica adequada, mas é de extrema importância o diagnóstico acurado no pré-operatório, quando pode ser constatado se a causa da baixa visão é realmente associada com a opacidade do cristalino e o acompanhamento pós-cirúrgico, feito até que se constate o restabelecimento da visão.

Avaliações do resultado visual dos pacientes operados são necessárias para se conhecer a repercussão das ações na comunidade, uma vez que permite que todos indivíduos operados, independente de qual o serviço que prestou o atendimento, seja avaliado, evitando o viés do cirurgião ser o avaliador do seu próprio resultado.

Os habitantes de uma região definida, moradores de cinco cidades localizadas na região centro-oeste do estado de São Paulo, foram avaliados quanto ao exame oftalmológico observado após facectomia.

É importante lembrar que na região de estudo há como hospital para atendimento SUS o HC da Faculdade de Medicina de Botucatu e que foram cadastradas várias clínicas particulares para a realização dos "mutirões" de catarata nos anos de 2005 e 2006. Portanto, os dados que foram levantados refletem os resultados das cirurgias feitas por estes serviços.

Dos pacientes avaliados, $66,7 \%$ tinham 70 anos ou mais e $55,3 \%$ eram do sexo feminino, semelhante ao observado em outros países $(75,4 \% \text { e } 69 \% \text { respectivamente) })^{(3)}$.

No presente estudo, houve melhora da AV após a refração em $29,7 \%$ dos casos. Muitos pacientes não possuíam correção óptica por falta de seguimento pós-operatório, ou seja, haveria melhores condições de AV em cerca de $30 \%$ dos indivíduos operados se os mesmos tivessem sido seguidos até o momento da prescrição de lentes corretoras.

Mesmo após a refração, cerca de $19 \%$ dos olhos operados permaneceram com $\mathrm{AV}<0,3$. Em estudos realizados em outros países em desenvolvimento, a AV foi menor que $0,3 \mathrm{em}$ $35,8 \% \%^{(4)}, 51,9 \%{ }^{(5)}$ e $24 \%{ }^{(6)}$.

Importante lembrar que em alguns países optou-se por deixar os indivíduos em condição de afacia, como em outro estudo, onde 49,4\% dos olhos permaneceram afácicos após

\begin{tabular}{|c|c|c|c|c|c|c|c|}
\hline \multirow{2}{*}{$\begin{array}{c}\text { Acuidade } \\
\text { visual }\end{array}$} & \multicolumn{7}{|c|}{ Diagnóstico } \\
\hline & $\begin{array}{l}\text { Astigmatismo } \\
\text { hipermetrópico }\end{array}$ & $\begin{array}{c}\text { Astigmatismo } \\
\text { miópico }\end{array}$ & $\begin{array}{c}\text { Astigmatismo } \\
\text { simples }\end{array}$ & Hipermetropia & Miopia & Emetropia & Total \\
\hline $0,3-0,7$ & 12 & 5 & 9 & 0 & 1 & 6 & 33 \\
\hline $0,05-0,3$ & 4 & 2 & 3 & 0 & 2 & 5 & 16 \\
\hline
\end{tabular}




\begin{tabular}{|c|c|c|c|c|c|c|c|c|}
\hline \multirow{2}{*}{$\begin{array}{l}\text { Acuidade } \\
\text { visual }\end{array}$} & \multicolumn{8}{|c|}{ Tratamento } \\
\hline & Nenhum & $\begin{array}{c}\text { Óculos com } \\
\text { AV normal }\end{array}$ & $\begin{array}{l}\text { Óculos com } \\
\text { deficiência } \\
\text { visual }\end{array}$ & $\begin{array}{l}\text { Mantido } \\
\text { óculos com } \\
\text { AV normal }\end{array}$ & $\begin{array}{c}\text { Mantido } \\
\text { óculos com } \\
\text { deficiência visual }\end{array}$ & $\begin{array}{l}\text { Não receitado } \\
\text { com deficiência } \\
\text { visual }\end{array}$ & Cegueira & Total \\
\hline$>0,7$ & 18 & 81 & 0 & 20 & 0 & 0 & 0 & 119 \\
\hline $0,3-0,7$ & 6 & 24 & 0 & 2 & 2 & 0 & 0 & 34 \\
\hline $0,05-0,3$ & 0 & 0 & 4 & 0 & 7 & 5 & 4 & 20 \\
\hline$<0,05$ & 0 & 0 & 2 & 0 & 0 & 1 & 13 & 16 \\
\hline \multirow[t]{2}{*}{ Total } & 24 & 105 & 6 & 22 & 9 & 6 & 17 & 189 \\
\hline & $(12,7 \%)$ & $(55,5 \%)$ & $(3,2 \%)$ & $(11,6 \%)$ & $(4,8 \%)$ & $(3,2 \%)$ & $(9,0 \%)$ & \\
\hline
\end{tabular}

extração extracapsular(7), o que resulta em baixa visual bastante importante. Os afácicos foram minoria no presente levantamento, ocorrendo em 7 (3,48\%) dos 201 olhos avaliados, aparentemente relacionados com sinais de complicações operatórias e não com escolha do cirurgião.

Nos locais onde a extração intracapsular foi mais freqüente, resultados piores foram encontrados ${ }^{(3-5)}$. A incidência de complicações cirúrgicas é mais comum na extração intracapsular $(14,5 \%)$ do que na extracapsular $(7,7 \%)^{(8)}$.

A baixa AV pode ser resultado de complicações cirúrgicas, o que foi encontrado em $8 \%$ (16) dos olhos avaliados, diferente da taxa encontrada em países desenvolvidos que é da ordem de $1 \%{ }^{(6)}$.

Além dos erros refrativos não corrigidos, como causa de baixa visão decorrente de complicação intra ou pós-operatória, a segunda causa foi opacidade de cápsula posterior, diferente do observado em outro estudo ${ }^{(8)}$, problema que seria facilmente solucionado se houvesse o seguimento do paciente e realização do Yag-laser.

Em 12 olhos (6\%), a presença de doença ocular concomitante foi a responsável pela baixa da AV. Os principais diagnósticos encontrados foram: cicatriz de coriorretinite, degeneração macular relacionada a idade, glaucoma e atrofia de papila. Apesar da avaliação do fundo-de-olho poder estar limitada quando a opacidade do cristalino é intensa ${ }^{(6)}$, uma boa avaliação ocular feita no pré-operatório livraria do estresse cirúrgico indivíduos para os quais a cirurgia não teve e não teria resultado positivo algum.

O presente estudo foi realizado em uma região que compreende muitos trabalhadores rurais. Em outros estudos, a baixa condição socioeconômica e o analfabetismo estiveram associados a piores resultados pós-operatórios ${ }^{(4-5)}$. Há maior dificuldade de acompanhamento, obtenção de medicação e correção óptica após a cirurgia. Além disso, há maior número de casos de cataratas hipermaduras tornando a extração da catarata mais trabalhosa.

Esta mesma constatação foi tirada de outro estudo, publicado no ano de 2002, no qual foram avaliados indivíduos de outra região do estado de São Paulo, quando $75,6 \%$ dos pacientes de uma localidade continuavam com AV abaixo de 0,2 após a cirurgia, sendo as principais causas de baixa visão os erros refrativos não corrigidos $(31,9 \%)$ e opacidade da cápsula posterior $(17,0 \%)$; em outra localidade daquela mesma região, $64,7 \%$ dos pacientes operados continuavam com baixa AV e as causas novamente eram opacidade de cápsula $(50,0 \%)$ e erro refracional $(9,0 \%)^{(9)}$.

Há que se chamar a atenção para que o número de cirurgias não deva ser mais importante que a qualidade destas ${ }^{(10)}$.

Sem dúvida, os projetos que permitem maior acesso da população ao tratamento cirúrgico da catarata, reduzem a cegueira pela catarata e devem ser estimulados.

Entretanto, há necessidade de avaliações detalhadas para indicação da cirurgia nos indivíduos que realmente dela necessitam e também seguimento longo e adequado, visando oferecer o atendimento integral ao paciente, inclusive com a prescrição óptica e o tratamento da opacidade capsular, causas já consagradas de baixa AV em facectomizados.

Novas campanhas de atendimento cirúrgico comunitário devem contemplar a avaliação da qualidade do serviço prestado, para que o indivíduo possa ser beneficiado pelos "mutirões" de catarata.

\section{CONCLUSÃO}

Após a facectomia $26,9 \%$ de indivíduos permanece com baixa visão. O presente estudo apontou como fatores de persistência da baixa AV em facectomizados os erros refrativos não corrigidos, opacidade de cápsula posterior, além de complicações operatórias e doenças nas quais não estaria indicado o ato operatório.

\section{ABSTRACT}

Purpose: To determine the outcomes and causes of visual impairment and blindness in cataract-operated patients who are living in the central-western zone of São Paulo state. Methods: A transversal, observational, systematic study was done involving patients inhabitants of five cities from a central-western zone of São Paulo state. The random sample was based on the Demographic Census Data (IBGE, 1995). All patients underwent to visual screening and complete eye examination. The indivi- 
duals who had cataract-operated eyes were separated for the present study. The results were statistically analyzed by descriptive methods, frequency of occurrence and concordance proportion with $95 \%$ confidence intervals. Results: At examination $2.37 \%$ of the subjects had cataract-operated eyes. Of the 201 operated eyes $26.9 \%$ had visual acuity compatible with blindness or visual impairment. The visual acuity persisted $<0.3$ even with spetacles in $19 \%$ of the patients. Twenty-seven and $9 \%$ of the patients had visual acuity improved with refractive error correction. The main causes of poor vision were refractive error, posterior capsule opacification (19.4\%), bullous keratophaty $(8.3 \%)$, cicatricial chorioretinitis $(8.3 \%)$, aphakic eyes $(8.3 \%)$, age-related macular degeneration $(5.5 \%)$, leukoma $(5.5 \%)$, glaucoma $(5.5 \%)$, optic atrophy $(5.5 \%)$, retinal detachment $(2.8 \%)$, retinal pigment epithelium atrophy $(2.8 \%)$ and high myopia (2.8\%). Conclusion: Cataract projects are effective in reducing preventable blindness caused by lens opacity. However, long-term scheduled evaluation of operated patients is necessary avoiding consequent blindness resulting from neglected follow-up.

Keywords: Cataract extraction; Health promotion; Blindness/prevention \& control; Vision, low; Populational surveys; Community health services

\section{REFERÊNCIAS}

1. World Health Organization. Regional Office for Europe. Vision 2020 [Internet] [cited 2007 Feb 20] Available from: www.euro.who.int/Document/RC56/ edoc05.pdf

2. Padilha MA. O direito de ver [Internet]. Rio de Janeiro: Sociedade Brasileira de Oftalmologia. [citado 2007 Jul 27]. Disponível em: http:// www.sboportal.org.br

3. Lau J, Michon JJ, Chan WS, Ellwein LB. Visual acuity and quality of life outcomes in cataract surgery patients in Hong Kong. Br J Ophthalmol. 2002; 86(1):12-7.

4. Bourne RR, Dineen BP, Ali SM, Huq DM, Johnson GJ. Outcomes of cataract surgery in Bangladesh; results from a population based nationwide survey. $\mathrm{Br}$ J Ophthalmol. 2003;87(7):813-7.

5. Dandona L, Dandona R, Naduvilath TJ, McCarty CA, Mandal P, Srinivas $\mathrm{M}$, et al. Population-based assessment of the outcome of cataract surgery in an urban population in southern India. Am J Ophthalmol. 1999;127(6):650-8.

6. Yamamoto N, Sugata Y, Murakami K, Yamamoto Y. Causes of blindness and the results of cataract surgical intervention in the Micronesian islands: a retrospective study. Int Ophthalmol. 2004;25(3):155-61.

7. Shrestha JK, Pradhan YM, Snellingen T. Outcomes of extracapsular surgery in eye camps of eastern Nepal. Br J Ophthalmol. 2001;85(6):648-52. Comment in: Br J Ophthalmol. 2001;85(6):635-7.

8. Prajna NV, Chandrakanth KS, Kim R, Narendran V, Selvakumar S, Rohini G, et al. The Madurai Intraocular Lens Study II: clinical outcomes. Am J Ophthalmol. 1998,125(1):14-25. Comment in: Am J Ophthalmol. 1999;127(1):110-1.

9. De Senne FM, Cardillo JA, Rocha EM, Kara-José N. Long-term visual outcomes in the Cataract-Free Zone Project in Brazil. Acta Ophthalmol Scand. 2002;80(3):262-6.

10. Johnson GJ. Improving outcome of cataract surgery in developing countries. Lancet. 2000,355(9199):158-9. Comment in: Lancet. 2000; 355(9199):180-4.

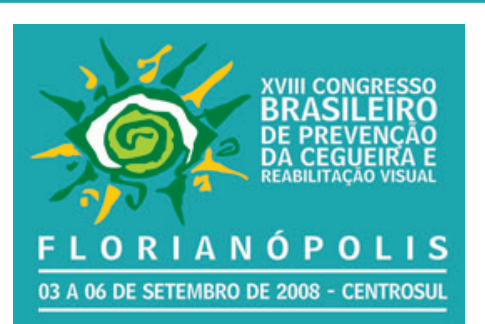

\section{Congresso Brasileiro de Prevenção da Cegueira e Reabilitação Visual}

\section{Último prazo para envio de trabalhos científicos (resumos)}

\section{$17 / 03 / 2008$}

\section{Só serão aceitos trabalhos enviados "online"}

\section{www.cbo2008.com.br}

